\title{
Interstitial high-dose-rate brachytherapy in the primary treatment of inoperable glioblastoma multiforme
}

\author{
Georgios Chatzikonstantinou, MD!, Peter Ulrich, MD², Eleftherios Archavlis, MD³, Nikolaos Zamboglou, MD!,4 \\ losif Strouthos, MD4, Eleni Zoga, MD5 , Dimos Baltas, MD,7, Nikolaos Tselis, MD' \\ 'Department of Radiotherapy and Oncology. University Hospital Frankfurt, Goethe University Frankfurt, Frankfurt am Main, Germany. \\ ${ }^{2}$ Krankenhaus Nordwest, Department of Orthopaedic and Spine Surgery, Section Neurosurgery. Frankfurt am Main, Germany, ${ }^{3}$ Department \\ of Neurosurgery, University Hospital Mainz, Johannes Gutenberg University, Mainz, Germany, ${ }^{4}$ German Oncology Center, Limassol, Cyprus, \\ ${ }^{5}$ Department of Radiation Oncology, Offenbach Hospital, Offenbach am Main, Germany, 'Division of Medical Physics, Department of \\ Radiation Oncology, Medical Center, Faculty of Medicine, University of Freiburg, Germany, ${ }^{7}$ German Cancer Consortium (DKTK) Partner Site \\ Freiburg, German Cancer Research Center (DKFZ), Heidelberg, Germany
}

\begin{abstract}
Purpose: To report our results of image-guided interstitial (IRT) high-dose-rate (HDR) brachytherapy (BRT) in the primary treatment of patients with inoperable glioblastoma multiforme (GBM) in the pre-temozolomide period.

Material and methods: Between 1994 and 2004, 17 patients were treated with HDR BRT for inoperable GBM. Of those, only 11 patients were treated with IRT BRT, and the remaining six patients received combined IRT BRT and external beam radiotherapy (EBRT). Patient's median age was 59.3 years (range, 29-83 years) and median tumor volume was $39.3 \mathrm{~cm}^{3}$ (range, 2-162 $\mathrm{cm}^{3}$ ). The prescribed HDR dose was median 40 Gy (range, 30-40 Gy), delivered twice daily in 5.0 Gy fractions over four consecutive days. Survival from BRT, toxicity as well as the impact of several prognostic factors was evaluated.

Results: At a median follow-up of 9.3 months, the median overall survival for the whole population, after BRT alone, and combined BRT with EBRT was 9.3, 7.3, and 10.1 months, respectively. Of the prognostic variables evaluated in univariate analysis, i.e., age, Karnofsky performance score, BRT dose, and tumor volume, only the latter one reached statistical significance. Two patients (11.7\%) developed treatment-associated adverse events, with one $(5.8 \%)$ symptomatic radionecrosis and one $(5.8 \%)$ severe convulsion episode, respectively.

Conclusions: For patients with inoperable GBM, IRT HDR BRT alone or in combination with EBRT is a safe and effective irradiation method providing palliation without excessive toxicity.

Key words: inoperable glioblastoma multiforme, high-dose-rate brachytherapy, external beam radiotherapy, combined irradiation, overall survival.

\section{Purpose}

While the treatment of operable glioblastoma multiforme (GBM) is well established through randomized clinical trials [1], the optimal therapeutic approach for primary inoperable disease is yet to be defined, with no standard recommended at present. In this clinical setting, management with best possible supportive care results in a median survival of approximately 3 months [2]. In inoperable patients that are clinically eligible for treatment, external beam radiotherapy (EBRT) [3], systemic chemotherapy (CHT) [4], or a combination of both $[5,6,7]$ are the most common utilized modalities prolonging survival up to 14 months. While the role of interstitial (IRT) brachytherapy (BRT) in the treatment of high-grade gliomas has been studied in the adjuvant [8] and recurrence setting [9], there are very few data concerning its implementation in the primary treatment of inoperable GBM $[10,11,12,13,14]$. In the present single-institute analysis, we report our experience with computed tomography (CT)-guided high-dose rate (HDR) BRT in the primary treatment of inoperable GBM in the pre-temozolomide (TMZ) era.

\section{Material and methods}

From 1994 to 2004, a total of 17 patients underwent HDR BRT alone or in combination with EBRT for inoperable GBM. Of those, six (37.5\%) patients underwent combined treatment, whereas the remaining eleven $(63.5 \%)$ patients were treated with sole BRT while refusing EBRT. Patients were judged inoperable by neurosurgical assess-
Address for correspondence: Georgios Chatzikonstantinou, Department of Radiotherapy and Oncology, University Hospital Frankfurt, J. W. Goethe University Frankfurt, Theodor-Stern-Kai 7, 60590 Frankfurt am Main, Germany, phone: +49-69-6301-5130, fax: +49-69-6301-5091,

هe-mail: Georgios.Chatzikonstantinou@kgu.de
Received: 10.12 .2018

Accepted: 02.04.2019

Published: 28.06.2019 
ment based on tumor location, lesion size, and performance status. No patient received CHT in conjunction with BRT or EBRT. Clinical judgment was used to define eligibility for BRT with patients fulfilling the following criteria: Karnofsky performance score (KPS) $\geq 50$, unilateral tumor growth involving no more than $1 / 3$ of the ventricles or corpus callosum, and maximal tumor diameter $\leq 10 \mathrm{~cm}$.

Table 1. Patient ( $n=17)$, tumor, and treatment characteristics

\begin{tabular}{|c|c|}
\hline Parameter & Results \\
\hline \multicolumn{2}{|l|}{ Age at diagnosis (years) } \\
\hline Median & 59.3 \\
\hline Range & $29-83$ \\
\hline \multicolumn{2}{|l|}{ KPS at BRT (\%) } \\
\hline Median & 90 \\
\hline Range & $50-100$ \\
\hline \multicolumn{2}{|l|}{ Initial EBRT dose (Gy) } \\
\hline Median & 60 \\
\hline Range & $40-60$ \\
\hline \multicolumn{2}{|l|}{ Time to BRT from diagnosis (months) } \\
\hline Median & 0.5 \\
\hline Range & $0-13$ \\
\hline \multicolumn{2}{|l|}{ Recurrent tumor volume at BRT $\left(\mathrm{cm}^{3}\right)$} \\
\hline Median & 39.3 \\
\hline Range & $2-162$ \\
\hline$<30 \mathrm{~cm}^{3}$ vs. $\geq 30 \mathrm{~cm}^{3}, n(\%)$ & $5(29.4)$ vs. $12(70.6)$ \\
\hline$<60 \mathrm{~cm}^{3}$ vs. $\geq 60 \mathrm{~cm}^{3}, n(\%)$ & 10 (58.8) vs. 7 (41.2) \\
\hline \multicolumn{2}{|l|}{ BRT dose (Gy) } \\
\hline Median & 40 \\
\hline Range & $30-40$ \\
\hline Surgery at diagnosis, $n$ (\%) & $0(0)$ \\
\hline Chemotherapy simultaneous to BRT, $n$ (\%) & $0(0)$ \\
\hline Chemotherapy simultaneous to EBRT, $n$ (\%) & $0(0)$ \\
\hline Surgery after BRT, $n$ (\%) & $1(5.8)$ \\
\hline For tumor progression & $0(0)$ \\
\hline For symptomatic radionecrosis & $1(5.8)$ \\
\hline EBRT after BRT & $0(0)$ \\
\hline Chemotherapy upon progression, $n(\%)$ & $2(11.7)$ \\
\hline Temozolomide & $1(5.8)$ \\
\hline Lomustin & $1(5.5)$ \\
\hline
\end{tabular}

Informed consent for the treatment was obtained from all patients. This retrospective analysis was approved by the local research ethics board. Epidemiological and clinical characteristics are summarized in Table 1.

\section{Treatment technique}

Our BRT technique has been described in detail elsewhere $[15,16]$. In short, the catheter implantation was performed with neurosurgical assistance using CT guidance under local anesthesia and sedoanalgesia, along with intravenous peri-interventional anticonvulsive/ anti-edematous prophylaxis. The implantation technique included transcranial insertion with the use of an acrylic template sutured to the scalp, with no requirement of a stereotactic frame (Figure 1). Positional control of the catheters was obtained by generating contrast-enhanced CT images with the catheters in situ under registration using a preinterventional contrast-enhanced magnetic resonance imaging (MRI). Thus, the maximum insertion depth, direction, and position of the catheters were estimated by interactive CT scanning. In addition, every catheter was marked at the template entry point with waterproof marker in order to detect possible geometrical changes during the fractionated treatment. In case of macroscopic deviations, the affected catheters were adapted to the initial depth position, and a new planning CT was performed to verify the accordance of the implant geometry with regard to the initial treatment plan. Treatment planning with anatomy-based three-dimensional (3D) dose optimization was conducted initially by Plato BPS (Nucletron, Veenendaal, The Netherlands), followed by Oncentra Brachy (Elekta, Veenendaal, The Netherlands). Gross tumor volume (GTV) was defined as the gadolinium-enhanced lesion in T1-weighted MRI images without the addition of further margins, thus planning target volume (PTV) corresponded to GTV. PTV coverage was defined as the proportion of PTV receiving at least the prescription dose defined as the average dose value on PTV surface, representing $100 \%$ isodose (Figure 2). Our HDR protocol delivered a total physical dose of $40 \mathrm{~Gy}$ in twice daily fractions of 5.0 Gy, over four con-

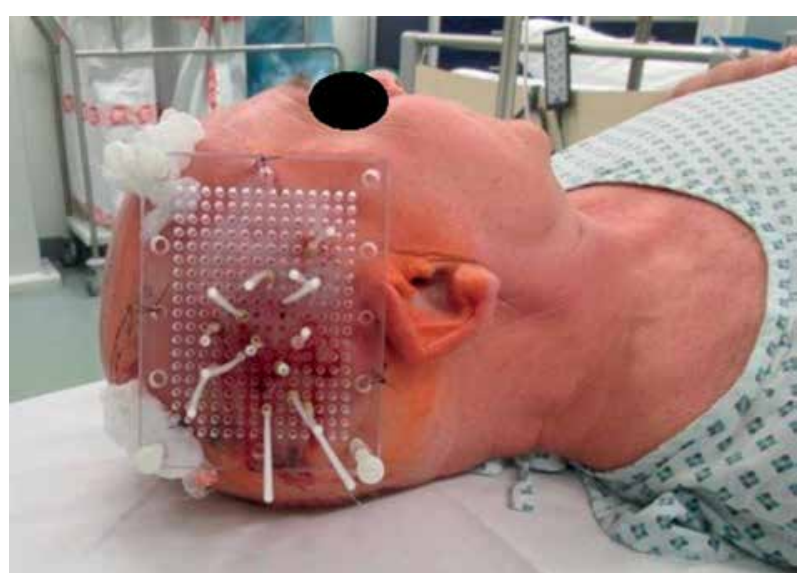

Fig. 1. Macroscopic template view with 14 implanted brachytherapy catheters. The template is sutured at the capillitium of the right temporal region 


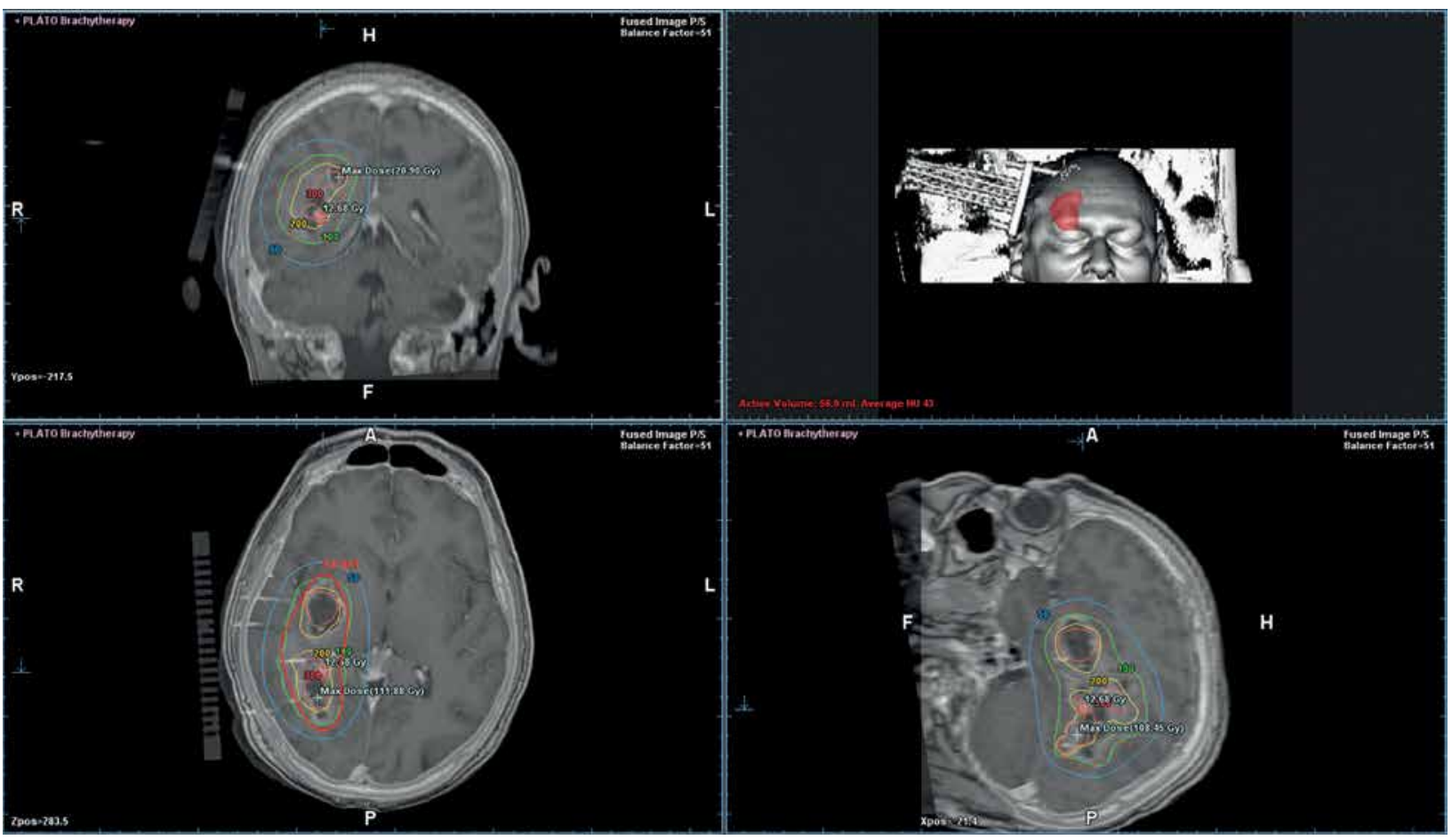

Fig. 2. Multiplanar three-dimensional view of the planning computed tomography data set of the same implant co-registered with a pretreatment magnetic resonance imaging data set with overlaid isodose distribution. The brachytherapy catheters are identifiable in situ. The volumetrically calculated lesion size amounted $58 \mathrm{~cm}^{3}$. The isodose lines colour code convention is: rose $=300 \%$ (isodose, $15.0 \mathrm{~Gy}$ ), yellow $=200 \%$ (isodose, $10.0 \mathrm{~Gy}$ ), green $=100 \%$ (isodose, $5.0 \mathrm{~Gy}$ ), light blue $=50 \%$ (isodose, $2.5 \mathrm{~Gy}$ ) per treatment fraction

secutive days, with an interfractional interval of at least 6 hours, which equals a biological effective dose of 65 Gy $(\mathrm{a} / \beta=8 \mathrm{~Gy})$ [17]. Catheters were removed after the last treatment fraction, and all irradiations were performed using an Iridium-192 HDR-afterloading system (micro Selectron-HDR, Nucletron).

\section{Follow-up}

Follow-up consisted of clinical and radiological evaluation (contrast-enhanced CT until 2001 and contrast-enhanced MRI ever since) performed initially at six weeks after BRT and every three months thereafter. Patients' data was collected from a prospectively maintained database and retrospective clinical chart review with data collection, allowing acquisition of information from referring neurosurgeons and radiation oncologists. Tumor response was assessed according to the Macdonald criteria [18]. Overall survival (OS) was calculated from initiation of BRT.

\section{Statistics}

Overall survival and treatment-related toxicity were the primary outcome measures considered for analysis. Univariate log-rank test was used to identify a possible correlation with following factors: median age, tumor volume, BRT dose, and KPS at the time of treatment. The estimated likelihood of events was calculated using the Kaplan-Meier method. A two-sided $p$ value $\leq 0.05$ was considered statistically significant. Statistical analysis was performed using the WinStat ${ }^{\circledR}$ software (R. Fitch Software, Bad Krozingen, Germany).

\section{Results}

\section{Oncological outcomes}

After a median follow-up of 9.3 months, the median OS for the whole population, after BRT alone, and

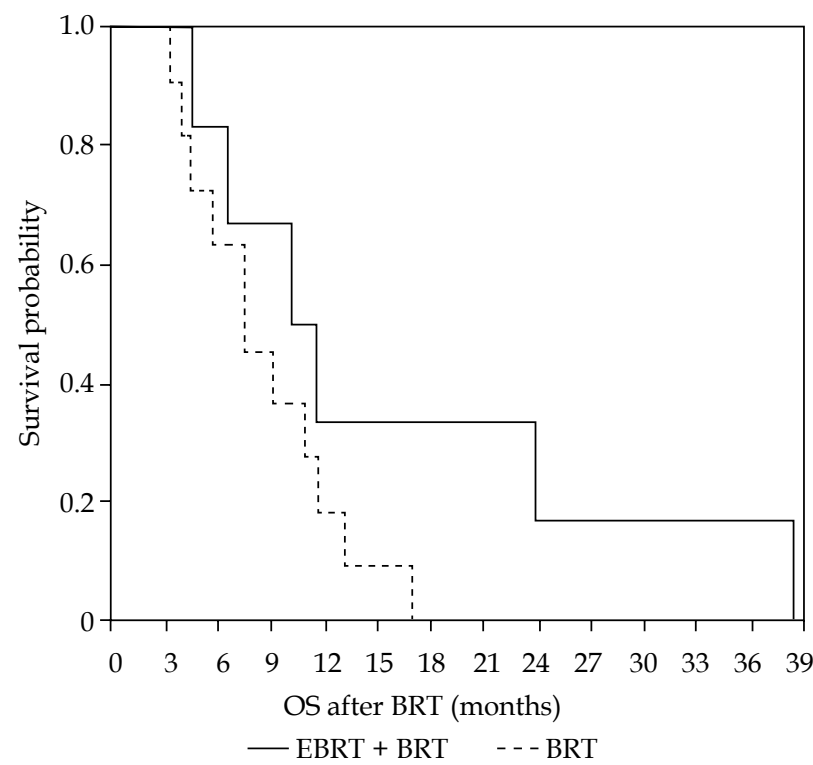

Fig. 3. Kaplan-Meier survival curves 
Table 2. Univariate results for survival after BRT

\begin{tabular}{|c|c|c|}
\hline Variable & $\begin{array}{l}\text { Survival after } \\
\text { BRT (months) }\end{array}$ & $P$-value \\
\hline \multicolumn{3}{|c|}{$\begin{array}{l}\text { Age (years) at BRT } \\
\text { (median, } 59.3 \text { years) }\end{array}$} \\
\hline$<59.3$ & 10.1 & 0.17 \\
\hline$\geq 59.3$ & 7.3 & \\
\hline \multicolumn{3}{|c|}{$\begin{array}{l}\text { Tumor volume at BRT }\left(\mathrm{cm}^{3}\right) \\
\left.\text { (median, } 39.3 \mathrm{~cm}^{3}\right)\end{array}$} \\
\hline$<39.3$ & 5.6 & 0.04 \\
\hline$\geq 39.3$ & 11.3 & \\
\hline \multicolumn{3}{|c|}{ BRT doses (Gy) } \\
\hline 30 & 6.5 & 0.71 \\
\hline 40 & 9.3 & \\
\hline \multicolumn{3}{|c|}{ KPS (\%) at BRT } \\
\hline$>80$ & 7.3 & 0.72 \\
\hline $70-80$ & 10.1 & \\
\hline$<70$ & 4.6 & \\
\hline
\end{tabular}

BRT-brachytherapy, KPS - Karnofsky performance score, EBRT-external beam radiation therapy

combined BRT with EBRT was 9.3, 7.3, and 10.1 months, respectively (Figure 3). Of the prognostic variables evaluated in univariate analysis, i.e., age, Karnofsky performance score, BRT dose, and tumor volume, only the latter reached statistical significance (Table 2).

\section{Toxicity}

Treatment was generally well tolerated. Two patients $(11.7 \%)$ developed treatment-associated adverse events, with one $(5.8 \%)$ case of symptomatic focal radionecrosis and one $(5.8 \%)$ severe convulsive episode. The median KPS of the entire patient population was 90 (range, 50-100) at the time of BRT. The corresponding median KPS at 6 weeks, 3 months, and 6 months after brachytherapy was 90 (range, 50-100), 90 (range, 50-100), and 80 (range, 50-100), respectively, suggesting no severe deterioration in the first 6 months following radiotherapy (RT). During hospitalization, all patients received anticonvulsive and anti-edematous treatment. With the exception of one patient experiencing symptomatic radionecrosis, anti-edem- atous treatment could be tapered off within three weeks after BRT treatment.

\section{Discussion}

Patients with inoperable primary GBM have a dismal prognosis, with no standard treatment currently recommended. The recently published National Cancer Database analysis [19] concerning the value of chemoradiotherapy (CRT) for elderly GBM patients in the TMZ era demonstrated that combined therapy is superior to both EBRT and CHT alone, even for patients undergoing biopsy only. Unfortunately, not all patients with unresectable GBM are eligible for combined CRT, particularly those with poor performance status, considering the aggressiveness of the approach. Colella et al. [7] reported on 24 patients with biopsy-proven only GBM who underwent primary CRT. Patients were treated with EBRT (60 Gy in 30 fractions) plus continuous daily TMZ (75 mg/m², 7 days per week), followed by six cycles of adjuvant TMZ $\left(200 \mathrm{mg} / \mathrm{m}^{2}\right)$. Survival analysis for the entire cohort showed a median survival of 13.8 months. At this, the effect of concurrent CRT compared to EBRT alone was analyzed by Kole et al. [20]. Using the National Cancer Database, the authors identified patients with histologically confirmed, biopsy only GBM who were treated from 2006-2011. The analysis included 1,479 patients among whom, $154(10.4 \%)$ received EBRT alone and 1,325 (89.6\%) concurrent CRT. The latter was associated with a significant OS benefit compared to sole EBRT (median 9.2 vs. 5.6 months, HR 0.64 [95\% CI: 0.54-0.76], $p<0.001)$, with the benefit persisting in multivariable (HR 0.71 [95\% CI: 0.60-0.85], $p<0.001$ ) and after propensity score-matched analysis.

Against this background, EBRT alone has been implemented in the treatment of inoperable GBM with results inferior to TMZ-based CRT. Beauchesne et al. [3] reported on the outcomes of a phase II study employing ultrafractionated EBRT comprised of three daily fractions of $0.75 \mathrm{~Gy}$, up to a total dose of $67.5 \mathrm{~Gy}$. Twenty-seven patients were treated, with $22(81.4 \%)$ completing the full course of RT and 16 (59.2\%) receiving further CHT for local disease progression. Overall survival was 9.5 months, with OS rates of $29 \%$ and $15 \%$ at 12 and 24 months, respectively. Treatment was well tolerated, with fatigue and alopecia grade II being the most frequently observed adverse events. To this end, data concerning the value

Table 3. Studies of interstitial brachytherapy in the treatment of inoperable primary glioblastoma multiforme

\begin{tabular}{|c|c|c|c|c|c|}
\hline Study & $\begin{array}{l}\text { Number } \\
\text { of patients }\end{array}$ & $\begin{array}{l}\text { Tumor volume } \\
\text { (median, } \mathrm{cm}^{3} \text { ) }\end{array}$ & Treatment & $\begin{array}{l}\text { Total dose } \\
\text { (median, Gy) }\end{array}$ & $\begin{array}{c}\text { Overall survival } \\
\text { (median, months) }\end{array}$ \\
\hline Julow et al. [10] & 3 & 12.5 & LDR & 60 & 7.6 \\
\hline Kickingereder et al. [11] & 103 & 15.5 & LDR & 60 & 11.1 \\
\hline Sparenberg et al. [14] & 16 & n.a. & HDR & 30 & 11.2 \\
\hline Micheletti et al. [13] & 17 & n.a. & HDR & $25 / 26.95$ & 8 \\
\hline Current study & 16 & 39.3 & $\mathrm{HDR}$ & 40 & 9.3 \\
\hline
\end{tabular}

LDR-low-dose-rate, n.a. - not available, HDR - high-dose-rate 
of BRT in the primary RT treatment of inoperable GBM are sparse $[10,11,13,14]$ (Table 3). Julow et al. [10] treated 10 patients with low-dose-rate (LDR) BRT, among which $3(30 \%)$ with inoperable and $7(70 \%)$ with recurrent GBM, respectively. Median dose was $60 \mathrm{~Gy}$ for a median tumor volume of $12.5 \mathrm{~cm}^{3}$. Median OS for the entire cohort was 7.6 months, with 1 -year OS rate of $17 \%$. In one of the largest series on IRT irradiation for GBM [11] on 201 patients, out of which 103 (51.2\%) were inoperable and 98 (48.8\%) with recurrent disease, received LDR BRT up to a total cumulative surface dose of median $60 \mathrm{~Gy}$. Furthermore, $90.3 \%$ received an additional EBRT boost (median dose, 25.2 Gy), whereas CHT was added to $30.8 \%$ of patients consisting of predominantly TMZ. Median tumor volume was 15.5 $\mathrm{cm}^{3}$. After a median follow-up of 9.8 months, median OS was 10.5 months with no significant differences among primary and recurrent tumors (11.1 vs. 10.4 months). For OS, multivariate analysis revealed Karnofsky performance score, age, and CHT as independent prognostic factors (all $p<0.01$ ). Transient and permanent toxicity was seen in $7.5 \%$ (15/201) and 2.0\% (4/201) of patients, respectively, with no procedure-related mortality. In contrary to LDR, HDR BRT is characterized by the delivery of high biological effective doses through hypofractionation, while ensuring maximized conformity through three-dimensional anatomy-oriented dose optimization [21]. Sparenberg et al. [14] reported on 38 patients including 16 with inoperable GBM, treated with combined HDR BRT and EBRT. The BRT protocol consisted of $30 \mathrm{~Gy}$ delivered over consecutive days at twice-daily fractions of $2.0 \mathrm{~Gy}$ with an interfractional interval of at least 6 hours. After 3 weeks, conventionally fractionated whole brain EBRT followed up to $30-40 \mathrm{~Gy}$. The mean OS for these 16 patients with inoperable disease was 11.2 months, with objectives $<40$ years and tumor volumes $<100 \mathrm{~cm}^{3}$, showing a significant better outcome. No symptomatic radionecrosis was documented. In a phase II study, Micheletti et al. [13] reported on 17 patients with inoperable high-grade gliomas undergoing primary HDR BRT. Interstitial RT was performed twice daily, with a single dose of either 5.0 Gy or 3.85 Gy over 5 or 7 fractions, respectively. Treatment was well tolerated with $2(8.3 \%)$ documented adverse events and a median OS of 8 months.

The current analysis represents one of very few clinical experiences on HDR BRT in the treatment of unresectable GBM. Although patients were treated in the pre-TMZ era, thus missing a valuable "tool" with proven efficacy on survival prolongation [22], the OS with combined HDR and EBRT was comparable to that of concurrent CRT and superior to EBRT alone [20]. Our data are corroborated by the results of Sparenberg et al. [14] who yielded an OS after combined HDR and EBRT of 11.2 months, while showing a positive correlation between outcome and tumor volume. The last factor was the only statistically significant parameter impacting the survival also in our study, yet with another cut-off. Considering the similar OS reported by Kickingereder et al. [11] using LDR BRT and CRT with $\mathrm{TMZ}$, one may theoretically argue that the addition of TMZ to HDR combined with EBRT could further improve the therapeutic ratio. The current analysis may serve as the first step towards exploration of this potential. In this light, a prospective trial is warranted in order to evaluate adequately the oncologic contribution of IRT HDR BRT as definitive treatment modality for inoperable primary GBM. Furthermore, an increasing volume of literature is communicating the impact of new systemic agents in the treatment of high-grade gliomas in the adjuvant and palliative setting $[23,24]$. None of our patients was treated with such substances. As such, a possible synergistic effect of HDR BRT in combination with newer systemic treatments could also be demonstrated in a prospective randomized setting.

\section{Conclusions}

Our data indicate that HDR BRT with or without EBRT is a safe and effective modality for the palliative treatment of inoperable GBM in selected patients. On that note, our data must be interpreted with caution being of retrospective nature, thus inherently susceptible to biases. Nevertheless, our encouraging results warrant a prospective evaluation in future studies in order to conclusively elucidate the role of HDR in the treatment of inoperable high-grade gliomas.

\section{Ethical approval}

An informed consent was obtained from every participant included in the study.

This was not a prospective randomized trial, but a retrospectively analyzed single institute cases series. All treatments were performed after written informed consent. The analysis of the treatment results was approved by ethical board of the hospital where the patients were treated.

\section{Disclosure}

Authors report no conflict of interest.

\section{References}

1. Stupp R, Mason WP, van den Bent et al. Radiotherapy plus concomitant and adjuvant temozolomide for glioblastoma. N Engl J Med 2005; 352: 987-996.

2. Prestwich RJ, Sivapalasunrtharam A, Johnston C et al. Survival in high-grade glioma: a study of survival in patients unfit for or declining radiotherapy. Clin Oncol 2005; 17: 133-137.

3. Beauchesne P, Bernier V, Carnin C et al. Prolonged survival for patients with newly diagnosed, inoperable glioblastoma with 3-times daily ultrafractionated radiation therapy. Neuro Oncol 2010; 12: 595-602.

4. Laigle-Donadey F, Figarella-Branger D, Chinot O et al. Upfront temozolomide in elderly patients with glioblastoma. J Neurooncol 2010; 99: 89-94.

5. Simon JM, Noël G, Chiras J et al. Radiotherapy and chemotherapy with or without carbogen and nicotinamide in inoperable biopsy-proven glioblastoma multiforme. Radiother Oncol 2003; 67: 45-51.

6. Barrié M, Couprie C, Dufour H et al. Temozolomide in combination with $\mathrm{BCNU}$ before and after radiotherapy in patients with inoperable newly diagnosed glioblastoma multiforme. Ann Oncol 2005; 16: 1177-1184. 
7. Colella M, Fiorica F, Api P et al. Radiochemotherapy for unresectable glioblastoma multiforme: a mono-institutional experience. WCRJ 2014; 1: e401.

8. Chang C, Chen W, Wei K et al. High-dose-rate stereotactic brachytherapy for patients with newly diagnosed glioblastoma multiformes. J Neurooncol 2003; 61: 45-55.

9. Tselis N, Kolotas C, Birn G et al. CT-guided interstitial HDR brachytherapy for recurrent glioblastoma multiforme. Longterm results. Strahlenther Onkol 2007; 183: 563-570.

10. Julow J, Viola A, Bálint $\mathrm{K}$ et al. Image fusion-guided stereotactic iodine-125 interstitial irradiation of inoperable and recurrent gliomas. Prog Neurol Surg 2007; 20: 303-311.

11. Kickingereder $P$, Hamisch $C$, Suchorska B et al. Low-dose rate stereotactic iodine- 125 brachytherapy for the treatment of inoperable primary and recurrent glioblastoma. Single-center experience with 201 cases. J Neurooncol 2014; 120: 615-623.

12. Sneed PK, Gutin PH, Prados MD et al. Brachytherapy of brain tumors. Stereotact Funct Neurosurg 1992; 59: 157-165.

13. Micheletti E, La Face B, Feroldi $\mathrm{P}$ et al. High-dose-rate brachytherapy for poor-prognosis, high-grade glioma: (phase II) preliminary results. Tumori 1996; 82: 339-344.

14. Sparenberg A, Ernst H. The value of fractionated afterloading therapy in the treatment of inoperable malignant brain tumor. Strahlenther Onkol 1990; 166: 251-255.

15. Kolotas C, Birn G, Baltas D et al. CT guided interstitial high dose rate brachytherapy for recurrent malignant gliomas. Br J Radiol 1999; 72: 805-808.

16. Chatzikonstantinou G, Zamboglou N, Archavlis E et al. CT-guided interstitial HDR-brachytherapy for recurrent glioblastoma multiforme: a 20-year single-institute experience. Strahlenther Onkol 2018; 194: 1171-1179.

17. Pedicini P, Fiorentino A, Simeon V et al. Clinical radiobiology of glioblastoma multiforme: estimation of tumor control probability from various radiotherapy fractionation schemes. Strahlenther Onkol 2014; 190: 925-932.

18. Macdonald DR, Cascino TL, Schold SC et al. Response criteria for phase II studies of supratentorial malignant glioma. J Clin Oncol 1990; 8: 1277-1280.

19. Rusthoven CG, Koshy M, Sher DJ et al. Combined-modality therapy with radiation and chemotherapy for elderly patients with glioblastoma in the temozolomide era: a national cancer database analysis. JAMA Neurol 2016; 73: 821-828.

20. Kole AJ, Park HS, Yeboa DN et al. Concurrent chemoradiotherapy versus radiotherapy alone for "biopsy-only" glioblastoma multiforme. Cancer 2016; 122: 2364-2370.

21. Milickovic N, Tselis N, Karagiannis E et al. Iridium-Knife: Another knife in radiation oncology. Brachytherapy 2017; 16: 884-892.

22. Perry JR, Laperriere N, O'Callaghan CJ et al. Short-course radiation plus temozolomide in elderly patients with glioblastoma. N Engl J Med 2017; 376: 1027-1037.

23. Mesti T, Ocvirk J. Malignant gliomas: old and new systemic treatment approaches. Radiol Oncol 2016; 50: 129-138.

24. Preusser M, Lim M, Hafler DA et al. Prospects of immune checkpoint modulators in the treatment of glioblastoma. Nat Rev Neurol 2015; 11: 504-514. 